\title{
FORMAÇÃO TÉCNICA EM PRÁTICAS INTEGRATIVAS E COMPLEMENTARES EM SAÚDE NA ESCOLA DE SAÚDE DA UFRN
}

\author{
M. M. S. ESTÁCIO*, A. C. V. V. AGUIAR, B. N. G. A. ROCHA e F. C. B. SILVA \\ Escola de Saúde da Universidade Federal do Rio Grande do Norte (ESUFRN) \\ merciaestacio@ig.com.br ${ }^{*}$
}

Artigo submetido em agosto/2015 e aceito em dezembro/2015

DOI: $10.15628 /$ rbept.2015.3344

\section{RESUMO}

Este texto apresenta o Curso Técnico em Práticas Integrativas e Complementares em Saúde (TPICS), oferecido pela Escola de Saúde da Universidade Federal do Rio Grande do Norte (ESUFRN), com a finalidade de analisar os objetivos propostos no seu Projeto Pedagógico, descrevendo como os mesmos estão sendo alcançados ao longo da sua trajetória. As terapias alternativas e complementares vêm sendo gradativamente inseridas no Sistema Único de Saúde, assim, emerge a necessidade de preparar o profissional técnico para atuar com um perfil voltado para terapêuticas mais acessíveis à população que surtam efeitos benéficos sem a exigência de utilizar recursos dispendiosos ao sistema de saúde. O curso TPICS encontra-se na sua sexta turma de alunos, e já formou 96 técnicos desde agosto de 2010. Durante a formação, os alunos desenvolvem, entre outras
\end{abstract}

competências, a noção de corporeidade no saber e fazer profissional para poder lidar com o holismo tão propagado nas políticas de saúde do Brasil, sendo estimulados a protagonizarem o processo de ensino e aprendizagem, estabelecendo uma relação crítica e progressiva ao longo do curso. A avaliação se dá de forma processual, dialógica e comprometida com o desenvolvimento pleno do aluno, nas dimensões humana, cognitiva, política, filosófica e ética. Contudo, observa-se que há a oferta de uma formação sólida considerando os princípios norteadores do Projeto Pedagógico do Curso a partir do pluralismo de ideias e concepções pedagógicas, agregando questões técnicas à formação crítica e humanista, fortalecendo a promoção da saúde através de abordagens integrais de cunho preventivista.

PALAVRAS-CHAVE: Terapias Complementares; Medicina Integrativa; Educação Profissionalizante; Educação Baseada em Competências.

\section{TECHNICAL TRAINING IN INTEGRATIVE AND COMPLEMENTARY PRACTICES IN HEALTH AT ESCOLA DE SAÚDE DA UFRN}

\begin{abstract}
This paper presents the Technical Course in Integrative and Complementary Practices in Health, offered by Escola de Saúde da Universidade Federal do Rio Grande do Norte, in order to analyze the proposed objectives of its Pedagogic Programme, describing how they are being achieved throughout its career. Alternative and complementary therapies have been gradually inserted into the National Health System thus emerges the need to prepare the technician to work applying profile facing to more accessible therapies to the population which rises beneficial effects that it don't need to use expensive resources to the health system. The mentioned course is in its sixth class students, and has trained 96 technicians since August 2010. During the training, students develop, among other powers, the notion
\end{abstract}

of corporeality in knowing and doing business to cope with holism as propagated in the health policies of Brazil, being encouraged to be protagonists in process of teaching and learning, establishing a critical and progressive relationship throughout the course. The evaluation takes place in a procedural, dialogical and committed way to the full development of student, in human, cognitive, political, philosophical and ethical dimensions. However, it is observed that there is offer of a solid education considering the guiding principles of the Pedagogical Project of the Course from the pluralism of ideas and pedagogical concepts, adding technical issues to critical and humanistic education, strengthening health promotion through integral approaches of preventivist nature.

KEYWORDS: Complementary Therapies; Integrative Medicine; Education, Professional; Competency-Based Education 


\section{INTRODUÇÃO}

O Curso Técnico em Práticas Integrativas e Complementares em Saúde (TPICS) é ofertado pela Escola de Saúde da Universidade Federal do Rio Grande do Norte (ESUFRN), desde agosto de 2010. Este curso é resultado de uma ampla discussão e reflexão entre docentes e discentes da ESUFRN. As inquietações diante da incorporação da Lei de Diretrizes e Bases da Educação (LDB) no 9.394/1996, Decreto Federal no 5.154/2004, do Parecer CNE/CEB no 11/2008 Cadastro e da Lei no 8.080/1990, SIS e Parecer no 353/1989 ampliaram o debate interno na ESUFRN e trabalhadores de instituições de saúde, objetivando operacionalizar de forma sistematizada as proposições dessas Leis e suas implicações para a formação técnica. O fruto desse processo apontou alguns caminhos para interseções lógicas e claras, dentro dos princípios da Educação Profissional que fundamentam o acesso amplo e democrático à educação de qualidade.

O curso se propõe a assegurar a formação de um profissional que atue no enfrentamento das questões presentes no cotidiano da rede de atenção à saúde, levando em consideração também o contexto externo, o qual influencia e é influenciado pela atuação destes mesmos profissionais.

O Projeto Pedagógico do Curso (PPC) estabelece estratégias pedagógicas que subsidiam o planejamento e a implementação de um currículo que fortaleça uma formação teórico-prática consistente e que assegure os princípios de capacitação voltados à habilitação de profissionais para o exercício das ações implementadas nas Políticas Nacional e Estadual de Práticas Integrativas e Complementares em Saúde (PNPIC e PEPIC) respectivamente, objetivando uma prestação de serviços com qualidade.

Nessa perspectiva, este curso se configura num desafio acadêmico de repensar a atuação dos trabalhadores no âmbito das práticas integrativas e complementares, consolidando a atuação destes profissionais na promoção e no cuidado em saúde. Este artigo se propõe a avaliar a trajetória do Curso Técnico em Práticas Integrativas e Complementares em Saúde (TPICS) e a analisar os objetivos do PPC, descrevendo como os mesmos estão sendo alcançados até o presente momento.

\section{DESENVOLVIMENTO}

\subsection{A construção do Curso Técnico em Práticas Integrativas e Complementares em Saúde}

A população brasileira sempre fez uso de terapias ditas alternativas e de cunho não invasivo. Esta necessidade de buscar apoio em outras terapêuticas, além da medicina convencional, sempre foi pautada em se oportunizar outros meios como forma de se obter melhora de quadros de males diversos à saúde.

As terapias alternativas e complementares vêm sendo gradativamente inseridas no Sistema Único de Saúde, como prevêem a Política Nacional de Promoção da Saúde, as Políticas Nacional e Estadual de Práticas Integrativas e Complementares (PNPIC, PEPIC) no SUS (Portarias Ministeriais no 687 de 30 de março de 2006, no 971 em 03 de maio de 2006, no 1.600, de 17 de julho de 2006 e no 154 de 24 de janeiro de 2008, no 274/GS, de 27 de junho de 2011.) 
A Política Nacional de Promoção da Saúde - PT no 687/GM, de 30 de março de 2006 -, compreende que:

As Práticas Corporais são expressões individuais e coletivas do movimento corporal advindo do conhecimento e da experiência em torno do jogo, da dança, do esporte, da luta, da ginástica. São possibilidades de organização, escolhas nos modos de relacionar-se com o corpo e de movimentar-se, que sejam compreendidas como benéficas à saúde de sujeitos e coletividades, incluindo as práticas de caminhadas e orientação para a realização de exercícios, e as práticas lúdicas, esportivas e terapêuticas, como: a capoeira, as danças, o Tai Chi Chuan, o Lien Chi, o Lian Gong, o Tui-ná, a Shantala, o Do-in, o Shiatsu, a Yoga, entre outras. Em face do caráter estratégico relacionado à qualidade de vida e à prevenção do adoecimento, as ações de Atividade Física/Práticas Corporais devem buscar a inclusão de toda a comunidade adstrita, não devendo restringir seu acesso apenas às populações já adoecidas ou mais vulneráveis. (BRASIL, 2006).

Estas políticas surgem fundamentadas nas premissas da Organização Mundial de Saúde (OMS) quanto à necessidade de se instalar nas instâncias dos serviços de saúde disponibilizados à população, principalmente na atenção básica, condutas terapêuticas que abarquem as práticas culturais utilizadas ao longo das suas vivências. Segundo a OMS o campo da PNPIC contempla sistemas médicos complexos e recursos terapêuticos, os quais são também denominados de Medicina Tradicional e Complementar/Alternativa (MT/MCA). No entanto, poucos profissionais integrantes da rede de serviços de saúde possuem algum, ou até nenhum preparo voltado para este universo terapêutico.

Diante do exposto, emerge a necessidade de preparar o profissional técnico com um perfil voltado para terapêuticas mais acessíveis à população, onde as práticas integrativas e complementares podem surtir efeitos benéficos sem a exigência de utilizar instrumentais ou recursos dispendiosos ao sistema de saúde ou à clientela assistida.

Atualmente, a ênfase dada à manutenção e a aparência do corpo advém de duas categorias básicas: a primeira refere-se a um corpo interior, o qual compreende a saúde e o seu funcionamento diante da doença e das deteriorações que acompanham o envelhecimento. A segunda refere-se à aparência, ao movimento e ao controle do corpo no espaço social. Ambas as categorias estão coligadas e determinam impressões nas imagens estilizadas da pós-modernidade.

Na cultura de consumo, o corpo é proclamado como um veículo de prazer, tornando-nos desejáveis de possuir corpos que se aproximam das imagens idealizadas de juventude, saúde, aptidões e beleza, para serem exibidos como valores de permuta. Nesta sociedade, a percepção do corpo é dominada por uma vasta ordem de imagens visuais que compõem a lógica interna do consumo.

O fato é que o discurso do corpo não escapa de nossa vida diária, mesmo quando considerado ausente, está por demais presente quando nos apropriamos de algo que nos sensibilize ao fato de que somos um corpo e temos um corpo. $O$ fato é que este oscila entre sujeito e objeto ${ }^{1}$ e se incorpora no cerne dos desejos sociais, próprios de uma cultura. "[...] A vida nos impõe o corpo cotidianamente, pois é nele e por ele que sentimos, desejamos, agimos e criamos"

\footnotetext{
${ }^{1}$ Em 1945, Merleau-Ponty aprofundou essa discussão em sua obra "Fenomenologia da Percepção".
} 
(VILLAÇA, GÓES, 1998, p. 23). Portanto, assumir que estamos vivos pressupõe uma condição carnal de um corpo que nos dá acesso ao mundo.

Assim, podemos afirmar que vivemos numa época em que se observa a grande necessidade das pessoas em serem atendidas com um olhar mais interior, ou seja, direcionando as ações de cuidado com o acolhimento e compreensão da subjetividade/objetividade do indivíduo.

Diante disso, a escolha por determinada abordagem, apoiada em uma compreensão da complexidade humana, que reconhece o indivíduo na sua essencialidade energética de emoções, sentimentos e vivências, requer do profissional de saúde uma visão de mundo estruturada nas energias que envolvem a vida, bem como as desarmonias decorrentes das experiências dos seres.

Atrelado a essa necessidade, os profissionais de saúde voltados à promoção da saúde na atualidade possuem pouco ou nenhum preparo em sua formação para lidar com essa visão do corpo, mente e espírito. A noção de corporeidade precisa ser inserida no saber e fazer do profissional de saúde para poder lidar com o tão almejado holismo que vem sendo ventilado nas políticas de saúde do Brasil.

O acesso a esse perfil formador, para o profissional técnico em práticas integrativas e complementares, além de promover conhecimento e sabedoria no universo subjetivo do ser humano, tende a facilitar a sua percepção frente àquele que é cuidado no seu contexto individual e coletivo. Assim, a população atendida poderá obter um atendimento individual e/ou coletivo onde seus desequilíbrios emocionais, espirituais, corpóreos e energéticos serão considerados em prol do equilíbrio necessário ao quadro em que a mesma se encontre.

Quando o profissional se dedica ao atendimento individual ou coletivo da clientela, na perspectiva holística, ele leva em consideração memórias de vida, metas a seguir e energia disponível. Para tanto, há necessidade de formação centrada em bases filosóficas que viabilizem conhecimento científico e empírico que proporcione meios para alcançar o saber e o fazer. Dessa forma, o curso técnico de práticas integrativas e complementares, contribui para o preparo de trabalhadores em saúde pautado nos princípios da integralidade, descentralização e participação.

Neste contexto, conhecendo a realidade do Rio Grande do Norte no tocante a necessidade de profissionais habilitados na área de PICS, a Escola de Saúde da UFRN, tem se preocupado em profissionalizar técnicos que desenvolvem atividades nesta área, suprindo esta lacuna, e contribuindo com os serviços de atenção e cuidado a saúde.

O curso Técnico em PICS contempla a organização dos conteúdos descritos em forma de competências, habilidades e bases tecnológicas, englobando módulos, núcleos, área e subáreas da saúde, constituídas de funções e subfunções/disciplinas específicas da formação técnica, com uma abordagem metodológica que pressupõe a interação do aluno com a realidade social.

A ESUFRN identifica a proposta do Curso Técnico em Práticas Integrativas e Complementares em Saúde, como um espaço de construção de conhecimentos e formação profissional. Trabalhando a formação do cidadão numa concepção de educação centrada em competências, preparando-o para o trabalho, sem, contudo, reduzir o processo educativo às flutuações do mercado. Adota, para tanto, o pluralismo de ideias e concepções pedagógicas, buscando conjugar questões técnicas com uma formação crítica e humanística, numa perspectiva de romper com padrões mecanicistas, possibilitando ao estudante uma melhor compreensão da sociedade e de suas diversidades. Vislumbra um profissional da área de saúde preparado para 
prestar um cuidado que atenda à integralidade da assistência à saúde como um direito de cidadania.

\subsection{Perfil profissional de conclusão dos egressos do curso}

O Curso Técnico em PICS se propõe a formar profissionais técnicos em saúde comprometidos com a consolidação das Políticas Nacional e Estadual de Práticas Integrativas e Complementares no SUS, por meio de situações de ensino-aprendizagem que propiciem o desenvolvimento das competências profissionais, anteriormente citadas, nas dimensões técnica, ética, política e comunicativa, para a melhoria da qualidade de vida da população. Nessa perspectiva almeja-se a construção de competências específicas para o profissional de nível técnico em PICS.

Considerando a finalidade de se profissionalizar para desenvolver práticas coletivas e individuais com ênfase na promoção do cuidado em saúde e na prevenção dos desequilíbrios energético-funcionais e/ou restauração das desarmonias existentes, espera-se que este profissional possua competências específicas para atuar na gestão do estresse, sendo capacitado a:

- Conduzir práticas corporais de descontração e relaxamento, advindas de tradições da medicina ayurvédica indiana, como o Yoga e da Medicina Tradicional Chinesa (MTC), como o Lian Gong;

- Aplicar técnicas de massagens relaxantes e estimulantes, do tipo terapêutica e estética;

- Utilizar técnicas oriundas da aromaterapia, cromoterapia, musicoterapia, auriculoterapia, moxabustão e ventosaterapia.

Aliado a isso, este profissional desenvolve competências gerais dos profissionais de nível técnico da área de saúde, a saber:

- Identificar os determinantes e condicionantes do processo saúde / doença;

- Identificar a estrutura e organização do sistema de saúde vigente;

- Identificar funções e responsabilidades dos membros da equipe de trabalho;

- Planejar e organizar o trabalho na perspectiva do atendimento integral e de qualidade;

- Realizar trabalho de equipe, correlacionando conhecimentos de várias áreas ou ciências, tendo em vista o caráter interdisciplinar;

- Aplicar as normas de biossegurança;

- Aplicar princípios e normas de higiene e saúde ambiental;

- Interpretar e aplicar legislação referente aos direitos do usuário;

- Identificar e aplicar princípios e normas de conservação de recursos não renováveis e de preservação do meio ambiente;

- Aplicar as normas de Saúde e Segurança do trabalho;

- Avaliar riscos de iatrogenias, ao executar procedimentos técnicos;

- Interpretar e aplicar normas do exercício profissional e princípios éticos que regem a conduta do profissional de saúde;

- Identificar e utilizar rotinas, protocolos de trabalho, instalações e equipamentos;

- Operar equipamentos próprios do campo de atuação, zelando pela sua manutenção;

- Registrar ocorrências e serviços prestados de acordo com exigências do campo de atuação; 
- Prestar informações aos usuários sobre os serviços que tenham sido prestados, de acordo com exigências do campo de atuação;

- Orientar os clientes adquirirem autonomia objetivando o autocuidado;

- Utilizar recursos e ferramentas de informática, específicos da área;

- Realizar primeiros socorros em situações de emergência.

Entendemos que a aquisição das habilidades específicas necessárias ao técnico em PICS, somada às competências gerais do profissional na área da saúde, fornecem subsídios concretos para uma atuação profissional comprometida com o cuidado e o gerenciamento do estresse.

\subsection{Avaliação}

A avaliação no curso técnico em PICS, é centrada no processo de ensino-aprendizagem e concebida como mais uma oportunidade de aprendizagem, na qual professor e aluno participam, acompanham e contribuem de maneira efetiva para a transformação da prática; uma avaliação compromissada com o desenvolvimento pleno do aluno, nas dimensões humana, cognitiva, política, filosófica e ética. Uma avaliação processual, dialógica, sem dia marcado para acontecer, porém acontecendo todo dia.

A legislação da educação profissional estabelece critérios de avaliação do desempenho do aluno sendo este considerado APTO, NÃO APTO, EM CURSO, INCOMPLETO² nas avaliações de desempenho parcial e final. Estes critérios norteiam docentes e discentes no julgamento das habilidades e competências a serem desenvolvidas durante todo o curso. A avaliação compreende fases que são complementares: avaliações parciais; avaliações de práticas e estágio curricular; e avaliação do desempenho final.

Ao aluno que apresenta dificuldades no domínio das competências e habilidades, são oportunizados, no decorrer do curso, trabalhos de recuperação e, se necessário, aulas de apoio. Os alunos podem recuperar suas faltas e/ou melhorar seu aproveitamento, ao longo do curso, desde que devidamente justificadas as faltas e planejado junto ao professor e à coordenação do curso.

Os alunos que, ainda assim, não são considerados aptos em uma competência no final, devem desenvolvê-la a partir de nova matrícula no semestre seguinte, de acordo com a sua oferta regular. Cabe aos professores do curso definirem a forma como o aluno adquirirá a competência: se este deverá cursar todo o módulo novamente, ou se receberá orientação específica do professor responsável, indicada na ata do Conselho de Curso final.

A frequência mínima exigida é de $75 \%$ do total das horas efetivamente trabalhadas pela escola, calculada sobre a totalidade dos componentes curriculares de cada módulo.

Será considerado concluinte do curso ou classificado para o módulo seguinte o aluno que tenha obtido aproveitamento suficiente para a aprovação (APTO) e a frequência mínima estabelecida, que terá apuração independente do aproveitamento.

\footnotetext{
2 APTO: O aluno desenvolveu as competências requeridas, com desempenho previsto; NÃO APTO: O aluno não desenvolveu as competências requeridas, com o desempenho desejado e/ou não cumpriu a frequência mínima exigida durante as aulas teórico-práticas ou estágio curricular; EM CURSO: $O$ aluno está cursando o módulo; INCOMPLETO: O aluno encontra-se em processo de recuperação de atividades e/ou estágios para desenvolvimento do desempenho desejado.
} 


\section{RESULTADOS E DISCUSSÕES}

A Escola de Saúde da UFRN tem como missão promover a profissionalização cidadã dos educandos para atuarem nos diversos níveis de atenção à saúde, de forma integral e crítica, considerando os princípios éticos, técnicos, políticos e humanos, na perspectiva de contribuir para a melhoria das condições de saúde da população. Nesse sentido, esta formação profissional está pautada em conhecimentos, habilidades e atitudes para apreensão, compreensão, análise e transformação da realidade, tanto no campo tecnológico como nos campos político, social, ético e humanístico.

No leque de opções de cursos técnicos oferecidos pela ESUFRN, está o Curso Técnico em Práticas Integrativas e Complementares em Saúde. Entendemos a importância de oferecer aos cidadãos tratamentos complementares de grande resolutividade, bem como de baixo custo, fator relevante principalmente para o atendimento oferecido na rede pública. Este curso encontra-se na sua sexta turma de alunos, e já formou 96 técnicos em PICS, desde a sua primeira oferta em agosto de 2010.

Diante das considerações tecidas anteriormente, onde elencamos as habilidades e objetivos de formação dos discentes, estabelecidos no Projeto Pedagógico do Curso (PPC) em PICS, acreditamos que estamos oferecendo uma formação sólida considerando os princípios norteadores, bem como, oportunizando condições de atuação na rede pública de saúde, na iniciativa privada ou de forma autônoma.

Estes fatos apontam para a necessidade de formação de um profissional qualificado não só para atender às demandas de um mercado globalizado e competitivo, mas, principalmente, preparado para tomar decisões diante de situações que requeiram habilidades e competências para o desenvolvimento do trabalho em saúde. A necessidade de capacitação desses profissionais decorre, inclusive, dos avanços tecnológicos e das profundas transformações econômicas e sociais pelas quais passa o mundo, neste início de milênio.

Com base nessa necessidade, estimulamos os alunos a serem protagonistas do seu processo de ensino e aprendizagem, solicitando registros que exigem a retomada dos conteúdos apresentados a cada módulo, estabelecendo uma relação crítica e progressiva ao longo do curso. Assim os alunos constroem seus portfólios apresentando os conhecimentos adquiridos mas, principalmente, se colocando de forma subjetiva diante deles, investindo no autoconhecimento e consequentemente na sua formação profissional.

\section{CONCLUSÃO}

As práticas elencadas na estrutura curricular do curso integram os conteúdos das terapêuticas mediante uma compreensão de corpo multidimensional e uma adoção de cuidados que amplia as possibilidades de encontros, observações, mobilização e escuta sensível para as pessoas adoecidas e saudáveis. A intenção, durante esse processo de promover saúde e cuidar do corpo, é construir relações de vínculo, de co-responsabilidade, inovadoras, autônomas e socialmente inclusivas, como propõe as Políticas Nacional e Estadual de Práticas Integrativas e Complementares em Saúde.

O curso adota o pluralismo de ideias e concepções pedagógicas, agrega questões técnicas à formação crítica e humanista, fora de padrões mecanicistas pela compreensão da sociedade na 
sua complexidade. Aposta num profissional em cujas atividades laborais e rotinas diárias, em unidades hospitalares e outros serviços, esteja presente o sentido de cuidar em saúde, pelo reconhecimento do contexto externo com suas influências, na relação entre quem cuida e quem é cuidado.

O empenho desejado assume relevância, visto que, haverá maior contingente de indivíduos capacitados para disseminar técnicas, hábitos e formas de conduzir os percalços da vida daqueles que são assistidos no cuidar. Dessa forma, torna-se possível ofertar de forma segura e responsável à população, as práticas integrativas e complementares como meio de fortalecer a promoção da saúde através de abordagens integrais de cunho preventivista.

\section{REFERÊNCIAS}

1. AGUIAR, A. C. V. V. Corporeidade e yoga: o transcender da educação para além do ego. Dissertaçao de mestrado. Programa de pós-graduação em Educação - UFRN, 2000.

2. ANTUNES, F. Conversa com dor. Rotary Club de Castelo Branco. Distrito 1960, 2002. Disponível em: < http://www.rotarycb.org/rotary/palestras/palestra_020917.html>. Acesso em: 26 set. 2008.

3. BARBOSA MA. A utilização de terapias alternativas por enfermeiros brasileiros. [tese]. São Paulo: Universidade de São Paulo, Escola de Enfermagem; 1994.

4. BRASIL. Ministério da Saúde. Secretaria-Executiva. Núcleo Técnico da Política Nacional de Humanização. HumanizaSUS: Política Nacional de Humanização: a humanização como eixo norteador das práticas de atenção e gestão em todas as instâncias do SUS. Brasília: Ministério da Saúde, 2004.

5. BRASIL. Ministério da Saúde. Portaria n. 687, de 30 de março de 2006. Dispõe sobre a Política Nacional de Promoção da Saúde no Sistema Único de Saúde. Brasília, 2006.

6. BRASIL. Ministério da Saúde. Secretaria de Atenção à Saúde. Departamento de Atenção Básica. Política Nacional de Práticas Integrativas e Complementares no SUS - PNPIC-SUS. Brasília: Ministério da Saúde, 2006a.

7. BRASIL. Ministério da Saúde. Conselho Nacional de Saúde. ATA da Centésima Sexagésima Segunda Reunião Ordinária do Conselho Nacional de Saúde - CNS. Brasília: Ministério da Saúde, 2006b.

8. BRASIL. Ministério da Saúde. Secretaria de Vigilância em Saúde. Secretaria de Atenção à Saúde. Política nacional de promoção da saúde. Brasília: Ministério da Saúde, 2006c.

9. BRASIL. Ministério da Saúde. Portaria n. 971, de 03 de maio de 2006. Dispõe sobre a Política Nacional de Práticas Integrativas e Complementares no Sistema Único de Saúde. Diário Oficial da União, Brasília, 4 maio 2006d.

10. BRASIL. Ministério da Saúde. Secretaria de Atenção à Saúde. Departamento de Atenção Básica. Política Nacional de Práticas Integrativas e Complementares no SUS - PNPIC-SUS. Brasília; 2006e.

11. BRASIL. Ministério da Saúde. Secretaria de Atenção à Saúde. Departamento de Atenção Básica. Política Nacional de Práticas Integrativas e Complementares no SUS - PNPIC-SUS atitude de ampliação de acesso. Brasília; 2008. 
12. BRASIL. Ministério da Saúde. Secretaria de Atenção à Saúde. Departamento de Atenção Básica. Práticas integrativas e complementares em saúde: uma realidade no SUS. Revista brasileira saúde da família. Brasília; 2008.

13. DAL MAS, W.D. Auriculoterapia: auriculomedicina na doutrina brasileira. Rio de Janeiro: Roca, 2005.

14. GEORGE JB. et al Teorias de Enfermagem. Porto Alegre: Artes Médicas; 1993.

15. GHAROTE, M. L. Técnicas de yoga. São Paulo: Phorte Editora, 2000.

16. GHAROTE, M. L. Yoga aplicada: da teoria à prática. São Paulo: Phorte Editora, 1996.

17. GOLA, O. J. Mapa auricular. São Paulo, 2002. Disponível em: <http://cseminario.vilabol.uol. com.br/mapauricular.htm>. Acesso em: 26 set. 2008.

18. HECKER, H.U. et al. Prática de Acupuntura. Rio de Janeiro: Guanabara Koogan, 2007.

19. VILLAÇA, N., GÓES, F. Em nome do corpo. Rio de Janeiro: Rocco, 1998.

20. INADA, T. Acupuntura e Moxabustão: uma coletânea e revisão sobre o tratamento de "cérvico/dorso/lombo/sacro/ciatalgia". São Paulo: Ícone, 2006.

21. KAGOTANI, Tsuguo. Shiatsu na estética. São Paulo: Andrei, 2004, 277p.

22. RIBEIRO, António Pinto. Por exemplo a cadeira: ensaios sobre as artes do corpo. Lisboa: Edições Cotovia, 1997.

23. ROSS, J. Combinações dos pontos de acupuntura: a chave para o êxito clínico. São Paulo: Roca, 2003.

24. SALLES, LF; FERREIRA, MZJ; SILVA MJP, TURRINI RNT. Terapias Complementares na Enfermagem: levantamento bibliográfico. Revista Nursing 2007; 105(9): 94-98.

25. SILVA, MJP; BENKO, MA. O uso das terapias alternativas por enfermeiros docentes. Rev. bras. enferm;51(3):457-68, jul.-set. 1998. tab.

26. VACCHIANO, Aridinéia. SHIATSU FACIAL: a arte do rejuvenescimento. 6ed. São Paulo: Ground, 2008, 177p. 